\title{
Nasopharyngeal Width and Its Association With Sleep-Disordered Breathing Symptoms in Children
}

\author{
Sang-Youp Lee (iD · Jeong-Whun Kim (i) \\ Department of Otorhinolaryngology-Head and Neck Surgery, Seoul National University Bundang Hospital, Seoul National University College of \\ Medicine, Seongnam, Korea
}

Objectives. Although adenotonsillar hypertrophy is the main cause of sleep-disordered breathing in children, surrounding anatomic factors, such as the width of the nasopharynx, can affect upper airway patency. However, there have been no reports of the association of nasopharyngeal width with sleep-disordered breathing in children. This study was undertaken to measure nasopharyngeal width in children undergoing adenotonsillectomy for sleep-disordered breathing and to investigate the clinical implications of this factor.

Methods. This was a retrospective study with a follow-up period of 1 year, performed at a tertiary referral center. We reviewed the operative records of children who underwent adenotonsillectomy at our center for symptoms of sleepdisordered breathing, such as snoring, apnea, and mouth breathing. The nasopharyngeal width was measured immediately before adenotonsillectomy, which was performed under general anesthesia with a microscopy-assisted mirror view. Adenotonsillar hypertrophy was graded on a four-point scale, and symptoms of sleep-disordered breathing were evaluated by using the Korean version of the Obstructive Sleep Apnea-18 questionnaire before and after surgery. The relationships between the average nasopharyngeal width and patient age and sex, adenotonsillar hypertrophy, and the Korean version of the Obstructive Sleep Apnea-18 score were analyzed.

Results. The study included 549 children (343 boys) with a mean age of 6.0 years (range, 2 to 11 years). The average nasopharyngeal width was $11.9 \mathrm{~mm}$ (range, 7.0 to $18.0 \mathrm{~mm}$ ) and increased with age (range, 11.2 to $13.3 ; \beta=0.264 ; P<$ 0.001 ). At 1 year after surgery, children with a greater nasopharyngeal width at the time of surgery exhibited additional improvements in symptoms of obstruction relative to those at 1 month after surgery.

Conclusion. The average nasopharyngeal width in children is approximately $11.9 \mathrm{~mm}$ and exhibits a slight increase with age. The width of the nasopharynx may be a factor associated with the degree of improvement in symptoms of sleepdisordered breathing after adenotonsillectomy.

Keywords. Sleep Apnea Syndromes; Snoring; Adenoidectomy; Tonsillectomy; Mouth Breathing; Retrospective Studies

\section{INTRODUCTION}

Otorhinolaryngologists often encounter children with symptoms of sleep-disordered breathing (SDB), such as snoring, sleep ap-

\footnotetext{
- Received August 5, 2018

Revised November 21, 2018

Accepted December 26, 2018

- Corresponding author: Jeong-Whun Kim

Department of Otorhinolaryngology-Head and Neck Surgery, Seoul National University Bundang Hospital, Seoul National University College of Medicine, 82 Gumi-ro 173beon-gil, Bundang-gu, Seongnam 13620, Korea

Tel: +82-31-787-7405, Fax: +82-31-787-4057

E-mail: kimemail@snubh.org
}

nea, and mouth breathing. Upper airway obstruction can occur in SDB at several levels of the upper airway, including the sinonasal tract, nasopharynx, oropharynx, hypopharynx, and larynx. Although any surrounding anatomic factor can affect the patency of the upper airway, adenotonsillar hypertrophy is the most common cause of upper airway obstruction in children [1]. The soft tissue of the nasopharynx grows rapidly at the age of 3-5 years although the area of the nasopharynx does not increase as rapidly during that time [2]. It is possible that the tonsils and adenoids grow more rapidly than the nasopharyngeal airway in children with SDB [3].

From the perspective of the anatomy of the nasopharynx, the

Copyright @ 2019 by Korean Society of Otorhinolaryngology-Head and Neck Surgery.

This is an open-access article distributed under the terms of the Creative Commons Attribution Non-Commercial License (http://creativecommons.org/licenses/by-nc/4.0)

which permits unrestricted non-commercial use, distribution, and reproduction in any medium, provided the original work is properly cited. 
nasopharyngeal airway is bordered anteriorly by the vomer and choanae, laterally by the torus tubarius and tubal tonsils, and posteriorly by the adenoids and cervical vertebrae [4]. Therefore, the patency at the level of nasopharynx can be determined by several soft tissue and skeletal factors, among which hypertrophy of the adenoids is the most common factor causing nasopharyngeal obstruction [5].

We have occasionally encountered children with a very narrow nasopharyngeal width at the time of surgery and wondered whether this small width could cause symptoms of upper airway obstruction. However, we could not identify any reports on the nasopharyngeal width in children, and, in particular, there is no information on the relationship between the nasopharyngeal width and patient age.

We hypothesized that the nasopharyngeal width may be one of the anatomic factors surrounding the upper airway that are associated with symptoms of SDB. The aims of this study were to obtain nasopharyngeal width data according to age and assess the relationship between this factor and symptoms of SDB in children undergoing adenotonsillectomy.

\section{MATERIALS AND METHODS}

\section{Study subjects}

We reviewed the operative records of children who underwent adenotonsillectomy for symptoms of SDB, such as snoring, apnea, and mouth breathing, from May 2011 through February 2017 at Seoul National University Bundang Hospital. Children from 2 to 11 years old were included. Children who had tonsillectomy without adenoidectomy, adenoidectomy without tonsillectomy, and revision surgery were excluded. And children who had congenital anomalies, syndromes, cerebral palsy, systemic disease, immunodeficiency disease, and central apnea were also excluded. Hypertrophy of the tonsils and adenoids was graded on a four-point scale [6,7]. The severity of SDB symptoms was evaluated using the Korean version of the Obstructive Sleep Apnea-18 questionnaire (KOSA-18) before and at 1 month and 1 year after surgery [8]. The study was approved by the Institutional Review Board of Seoul National University Bundang

\section{H I G G H L I I G H T S}

- This study assessed nasopharyngeal width in children with sleep-disordered breathing.

- Nasopharyngeal width was measured before adenotonsillectomy under general anesthesia.

- Greater nasopharyngeal width was linked with long-term improvements in symptoms of obstruction.

- Nasopharynx width may be associated with improvement in sleep-disordered breathing after adenotonsillectomy.
Hospital (IRB No. B-1709-418-103). The need for informed consent was waived in view of the retrospective nature of the research and the anonymity of the data.

\section{Grading scale for tonsils and adenoids}

Tonsillar hypertrophy was evaluated using the Brodsky grading scale as follows: grade 0 , no tonsil tissue present; grade 1 , tonsils account for $<25 \%$ of the airway area; grade $2,25 \%$ to $50 \%$ of the airway area; grade $3,50 \%$ to $75 \%$ of the airway area; and grade $4,>75 \%$ of the airway area $[6,9,10]$. Adenoid hypertrophy was evaluated using the modified Josephson grading scale as follows: grade 0 , no adenoid tissue present; grade 1 , adenoids account for $<25 \%$ of the airway area; grade $2,25 \%$ to $50 \%$ of the airway area; grade $3,50 \%$ to $75 \%$ of the airway area; and grade $4,>75 \%$ of the airway area $[7,11]$.

\section{KOSA-18 questionnaire}

The KOSA-18 questionnaire is used to evaluate the quality of life in children with obstructive sleep apnea. It comprises 18 items, and each item is graded on a seven-point scale depending on its severity. In the present study, the total KOSA-18 score and individual scores for snoring, apnea, and mouth breathing were calculated for patients.

\section{Measurement of the nasopharyngeal width}

The width of the nasopharynx was defined as the smallest distance between the medial sides of the right and left torus tubarius. It was measured meticulously just before adenotonsillectomy under general anesthesia and a microscopy-assisted mirror view (Fig. 1). When the adenoid was severely hypertrophic, we removed some protruding part of the adenoid until measure-

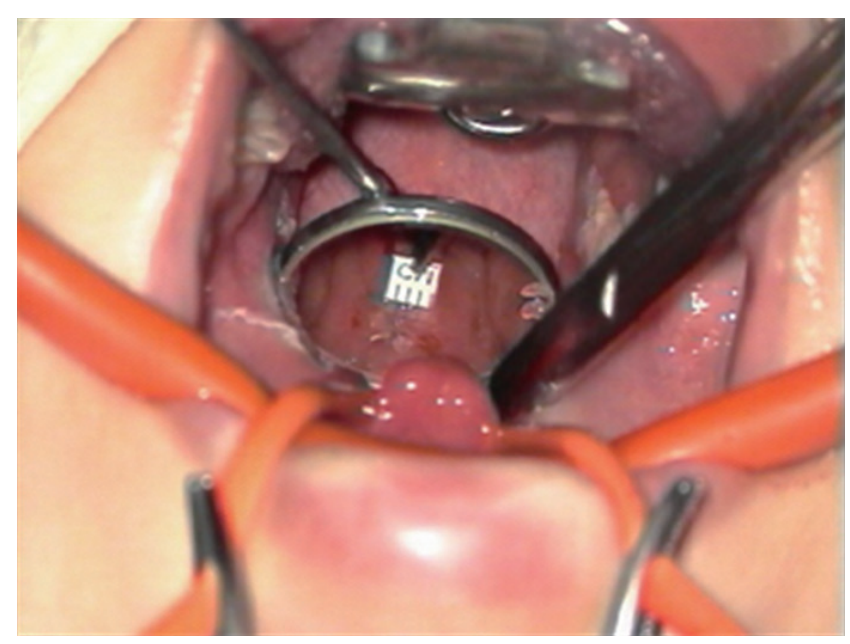

Fig. 1. Assessment of the nasopharyngeal width before adenotonsillectomy in children with sleep-disordered breathing. The width of the nasopharynx is measured as the distance between the medial sides of the left and right torus tubarius. The ruler is calibrated in millimeters. 
ment was feasible to measure the nasopharyngeal width accurately. To investigate the relationship between the nasopharyngeal width and improvements in symptoms of SDB, we divided the children into two subgroups according to the width of the nasopharynx. To control for potential age-related effects, we defined the nasopharyngeal width as "narrower" if it was less than the average value for the same age group and "wider" if it was equal to or greater than the average value for the same age group.

\section{Statistical analysis}

The results are presented as mean \pm standard deviation. Multivariate linear regression analysis was performed to investigate the relationship between the nasopharyngeal width and age, sex, the tonsil grade, the adenoid grade, and the KOSA-18 score. The severity of SDB symptoms at 1 month and 1 year after surgery was compared with that at baseline using a paired $t$-test. We used data of all 549 subjects in analyzing the relationship between nasopharyngeal width and age, sex, the tonsil grade, the adenoid grade, and the KOSA-18 score. However, we used data of only 133 subjects in analyzing the severity of SDB symptoms because 413 subjects did not have KOSA-18 questionnaire completely. All statistical analyses were performed using IBM SPSS ver. 22.0 (IBM Corp., Armonk, NY, USA). A $P$-value of $<0.05$ was considered statistically significant.

\section{RESULTS}

A total of 549 children (including 343 boys) with a mean age of 6 years (range, 2 to 11 years) were included. Most age groups included more boys than girls, with a male to female ratio of approximately 1.7 to 1.0 (Table 1 ).

\section{Tonsil and adenoid grades}

The distribution of tonsil grades was as follows: grade $1, n=0$; grade 2, $n=58$; grade $3, n=212$; grade $4, n=279$ (Fig. $2 \mathrm{~A}$ ). The

Table 1. Distribution of children undergoing adenotonsillectomy for sleep-disordered breathing and the nasopharyngeal width according to age

\begin{tabular}{cccc}
\hline Age $(\mathrm{yr})$ & No. of patient & Male:female & Nasopharyngeal width $(\mathrm{mm})$ \\
\hline 2 & 5 & $3: 2$ & $11.2 \pm 1.3(10.0-13.0)$ \\
3 & 43 & $28: 15$ & $11.1 \pm 1.6(7.5-15.0)$ \\
4 & 94 & $68: 26$ & $11.3 \pm 1.6(7.0-15.0)$ \\
5 & 114 & $78: 36$ & $11.3 \pm 1.6(8.0-15.0)$ \\
6 & 103 & $56: 47$ & $12.0 \pm 1.5(8.0-16.0)$ \\
7 & 72 & $37: 35$ & $12.0 \pm 1.7(8.0-16.0)$ \\
8 & 58 & $39: 19$ & $12.2 \pm 1.8(8.0-16.0)$ \\
9 & 27 & $13: 14$ & $12.6 \pm 1.9(8.0-17.0)$ \\
10 & 31 & $20: 11$ & $13.0 \pm 2.0(9.0-18.0)$ \\
11 & 20 & $15: 5$ & $13.3 \pm 2.0(7.0-16.0)$ \\
Total & 567 & $357: 210$ & $11.8 \pm 1.7(7.0-18.0)$ \\
\hline
\end{tabular}

Values are presented as mean \pm standard deviation (range). distribution of adenoid grades was as follows: grade $1, \mathrm{n}=28$; grade 2, $n=145$; grade 3, $n=203$; and grade $4, n=171$ (Fig. 2B).

\section{Nasopharyngeal width}

The average nasopharyngeal width was $11.9 \mathrm{~mm}$ (range, 7.0 to $18.0 \mathrm{~mm}$ ), and it increased with increasing age (Fig. 3), from an average of $11.2 \mathrm{~mm}$ at the age of 3 years to $13.3 \mathrm{~mm}$ at 11 years (Table 1). Multivariate regression analysis revealed a significant association of the nasopharyngeal width with age $(\beta=0.264, P<$ $0.001)$, but not with sex, age, the tonsil grade, the adenoid grade, and the KOSA-18 score.

\section{The relationship between tonsil or adenoid grades and nasopharyngeal width}

A linear correlation between tonsil grades and adenoid grades ( $r=-0.170, P<0.001$; adjusted by sex, age, and nasopharyngeal width) was identified (adjusted by sex, age, and nasopharyngeal width). However, no correlation between tonsil grades and nasopharyngeal width was identified $(P=0.573$; adjusted by sex, age, and adenoid grades). Also, there was no correlation between adenoid grades and nasopharyngeal width was identified $(P=$ 0.756; adjusted by sex, age, and tonsil grades).
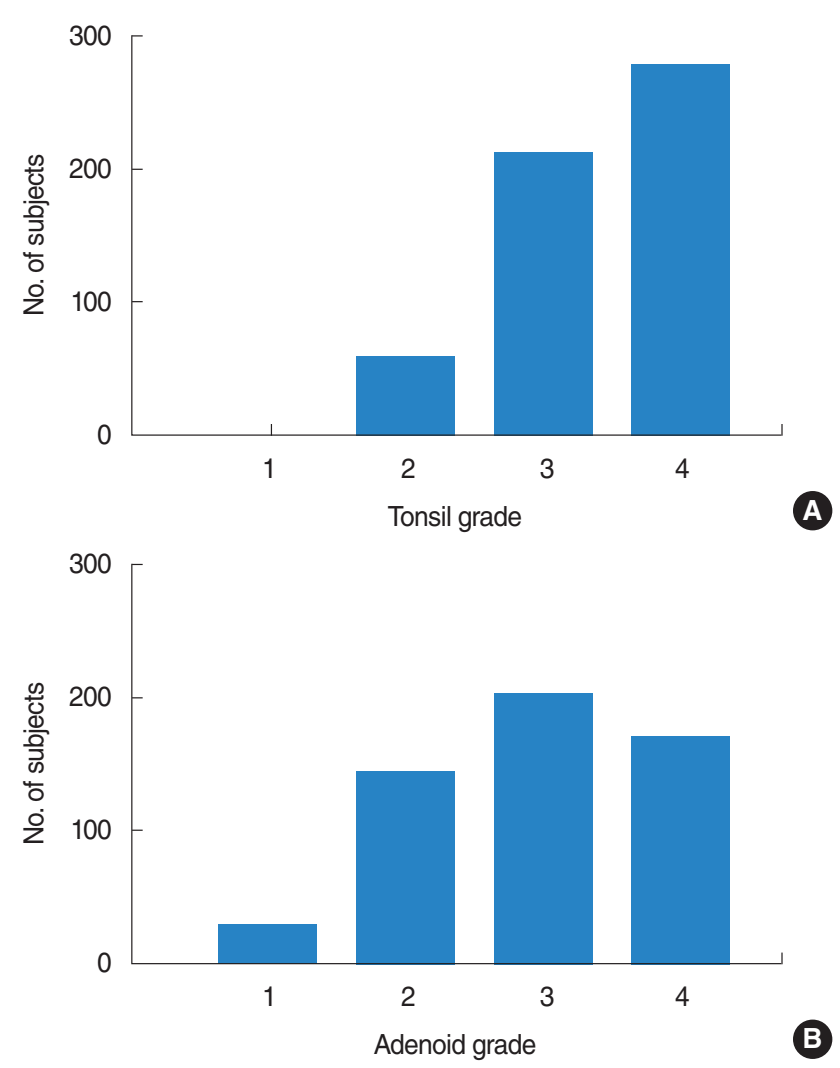

Fig. 2. Distribution of tonsil (A) and adenoid (B) grades in children undergoing adenotonsillectomy for sleep-disordered breathing. Tonsillar hypertrophy was evaluated using the Brodsky grading scale, while adenoid hypertrophy was evaluated using the modified Josephson grading scale. 


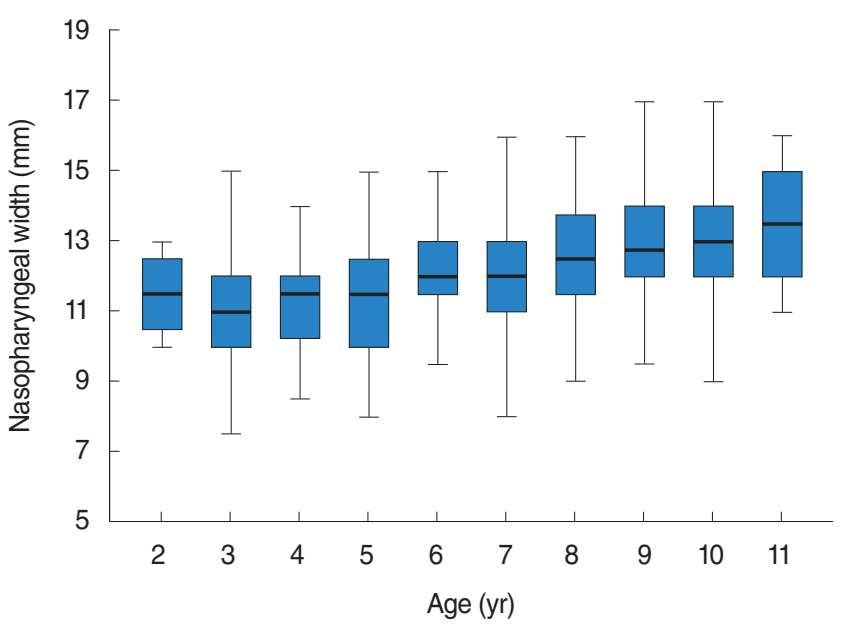

Fig. 3. Distribution of nasopharyngeal width by age. The figure shows that the nasopharyngeal width of children with sleep-disordered breathing tends to increase with age.

\section{Effect of the nasopharyngeal width on long-term improvements in SDB symptoms}

Postoperative 1-month and 1-year KOSA-18 scores were compared between the wider and narrower nasopharyngeal width groups in order to investigate whether the nasopharyngeal width at the time of adenotonsillectomy influenced long-term improvements in SDB symptoms. Because of missing data in KOSA-18, the comparison was performed in 57 subjects in the narrower nasopharynx group, and 76 subjects in the wider nasopharynx group. No statistical differences in age, tonsil grade, and adenoid grade between group of "narrower nasopharynx" and "wider nasopharynx" were identified (Supplementary Table 1). There was no significant difference in the preoperative KOSA-18 score between the two groups. While the 1-month postoperative KOSA18 scores were significantly improved in comparison with the preoperative scores in both groups, the total KOSA-18 score and the individual scores for snoring, apnea, and mouth breathing exhibited additional improvements at 1 year only in children with a greater nasopharyngeal width at the time of surgery $(P=0.027$ for total KOSA-18 score, $P=0.021$ for snoring, apnea, and mouth breathing scores). There was no significant additional improvement at 1 year in children with a smaller nasopharyngeal width (Table 2). When postoperative 1-month and 1-year KOSA-18 scores were compared in each tonsil grade, no statistically significant improvement was identified (Supplementary Table 2). When the same comparisons were done for each adenoid grade, there are some statistically significant correlations for total KOSA-18 score (Supplementary Table 3). However, those were validated only in adenoid grade 1 and 4 . No correlation between nasopharyngeal width and subitems (snoring, apnea, and mouth breathing) was identified.
Table 2. Comparison of upper airway obstruction symptoms at 1 month and 1 year after adenotonsillectomy in children with sleepdisordered breathing

\begin{tabular}{|c|c|c|c|c|}
\hline \multirow{2}{*}{$\begin{array}{l}\text { Time of postoperative } \\
\text { assessment }\end{array}$} & \multicolumn{2}{|c|}{$\begin{array}{l}\text { Narrower nasopharynx } \\
\qquad(\mathrm{n}=60)\end{array}$} & \multicolumn{2}{|c|}{$\begin{array}{l}\text { Wider nasopharynx } \\
\qquad(\mathrm{n}=76)\end{array}$} \\
\hline & Mean $\pm S D$ & $P$-value & Mean \pm SD & $P$-value* \\
\hline Total KOSA-18 score & & 0.288 & & 0.027 \\
\hline 1 Month & $35.7 \pm 11.7$ & & $36.7 \pm 11.7$ & \\
\hline 1 Year & $34.1 \pm 11.6$ & & $33.7 \pm 10.5$ & \\
\hline Snoring score & & 0.792 & & 0.021 \\
\hline 1 Month & $1.7 \pm 0.8$ & & $1.9 \pm 1.2$ & \\
\hline 1 Year & $1.6 \pm 0.7$ & & $1.6 \pm 0.7$ & \\
\hline Apnea score & & 0.792 & & 0.021 \\
\hline 1 Month & $1.7 \pm 0.8$ & & $1.9 \pm 1.2$ & \\
\hline 1 Year & $1.6 \pm 0.7$ & & $1.6 \pm 0.7$ & \\
\hline Mouth breathing score & & 0.792 & & 0.021 \\
\hline 1 Month & $1.7 \pm 0.8$ & & $1.9 \pm 1.2$ & \\
\hline 1 Year & $1.6 \pm 0.7$ & & $1.6 \pm 0.7$ & \\
\hline
\end{tabular}

SD, standard deviation; KOSA-18, Korean version of the Obstructive Sleep Apnea-18 questionnaire. ${ }^{*} P<0.05$.

\section{DISCUSSION}

Most studies on SDB in children have focused on the amount of enlarged lymphoid tissue. It is well-known that the size of the tonsils and adenoids is positively correlated with the severity of pediatric SDB [12]. However, the size of the tonsils and adenoids cannot explain all aspects of SDB in children. Other studies have focused on the size of the airway rather than hypertrophy of the lymphoid tissue [12-16]. In one of these studies, the pediatric SDB index was negatively correlated with the volume of the oropharynx [12]. Another study showed that children with SDB had a smaller cross-sectional total airway area and volume on three-dimensional radiographs [14].

We used a simple method to measure the nasopharyngeal airway space as one of the factors associated with upper airway obstruction in children with SDB. We hypothesized that the nasopharyngeal width would be a good indicator of the abovementioned nasopharyngeal airway space. We defined this width as the narrowest distance between the medial sides of the right and left torus tubarius. No studies have investigated the nasopharyngeal width in pediatric patients with SDB. In addition, no studies have attempted to analyze the nasopharyngeal width as one of the factors associated with symptoms of SDB. To the best of our knowledge, this is the first study to analyze the nasopharyngeal width in children.

When we measured the nasopharyngeal width directly, the average width in children was $11.9 \mathrm{~mm}$ (range, 7.0 to $18.0 \mathrm{~mm}$ ). Using multivariate regression analysis, we investigated the association between the nasopharyngeal width and other variables, including age, sex, the tonsil grade, the adenoid grade, and the total KOSA-18 symptom score. Of these factors, only age was 
positively associated with the nasopharyngeal width. However, the increase in width was only $0.26 \mathrm{~mm}$ per year. The minimum value in the 2-year age group was remarkably larger than that in the other age groups because the number of patients in this group was limited and, consequently, the probability of outliers was low.

In subgroup analyses performed after the children were divided according to the nasopharyngeal width, we found additional long-term improvements in SDB symptoms only in children with a greater nasopharyngeal width. Therefore, we assume that the nasopharyngeal width may be an important component of the nasopharyngeal airspace, and that it has a significant association with long-term improvements in SDB symptoms after adenotonsillectomy.

According to previous studies, there are several factors that may influence the resolution of a patient's symptoms after adenotonsillectomy. Some researchers have reported that obesity at diagnosis and the severity of SDB are important determinants of the surgical outcome [17-19]. Symptoms have been reported to persist in $20 \%-75 \%$ patients who undergo adenotonsillectomy, and the risk was found to be high in obese patients and those with a high apnea-hypopnea index value [17-19]. One study showed that symptoms were less likely to improve in patients with a narrower epipharyngeal airspace after adenotonsillectomy [20]. This is consistent with our finding that the prognosis may be more favorable if the nasopharyngeal width is greater. The abovementioned study also suggested that an abnormal facial morphology is one of the factors that may affect the surgical outcome [20]. Similarly, another study reported that craniofacial disproportion, hypotonia, and redundant soft tissue in the oropharynx were associated with persistent symptoms of SDB after adenotonsillectomy [21,22].

Our study has some limitations. First, it only included children who underwent adenotonsillectomy for SDB symptoms associated with hypertrophy of the tonsils or adenoids; normal controls with SDB were not included. However, considering that the tonsil and adenoid grades were not associated with the nasopharyngeal width, the nasopharyngeal width of normal controls may not be significantly different from that of the patients in our study. Second, because we set only KOSA-18 data as dependent variable about long term improvement, we could not analyze objective results. To do objective analysis, we should have performed polysomnography. Third, there were a lot of missing data in KOSA-18, it could be a cause of statistical error. However, we believe that our study is meaningful in that it provides the first reference range for the nasopharyngeal width in children and highlights the clinical implications in terms of the likelihood of long-term improvements in symptoms of SDB after adenotonsillectomy.

In this study, the average nasopharyngeal width in children was approximately $12 \mathrm{~mm}$, and it exhibited a slight increase with increasing age. The width of the nasopharynx may be one of the determinants of the extent of improvements in SDB symptoms after adenotonsillectomy; additional symptomatic improvements at 1 year after surgery were found only in children with a greater nasopharyngeal width at the time of surgery. Further studies are warranted to identify whether the nasopharyngeal width during childhood is associated with the development of obstructive sleep apnea in adults.

\section{CONFLICT OF INTEREST}

No potential conflict of interest relevant to this article was reported.

\section{ACKNOWLEDGMENTS}

This research was partly supported by the Bio and MedicalTechnology Development Program of the National Research Foundation (NRF) funded by the Korean Government, Ministry of Science and ICT, Sejong, Korea (NRF-2015M3A9D7066972, NRF2015M3A9D7066973).

\section{ORCID}

Sang-Youp Lee https://orcid.org/0000-0002-3031-8787

Jeong-Whun Kim https://orcid.org/0000-0003-4858-3316

\section{AUTHOR CONTRIBUTIONS}

Conceptualization: JWK. Data curation, Formal analysis, Methodology, Project administration: all authors. Writing - original draft: SYL.Writing - review \& editing: JWK.

\section{SUPPLEMENTARY MATERIALS}

Supplementary materials can be available at https://doi.org/10. 21053/ceo.2018.01151.

\section{REFERENCES}

1. Simsek G, Karacayli C, Ozel A, Arslan B, Muluk NB, Kilic R. Blood parameters as indicators of upper airway obstruction in children with adenoid or adenotonsillar hypertrophy. J Craniofac Surg. 2015 May; 26(3):e213-6.

2. JeansWD, Fernando DC, Maw AR, Leighton BC.A longitudinal study of the growth of the nasopharynx and its contents in normal children. Br J Radiol. 1981 Feb;54(638):117-21.

3. Papaioannou G, Kambas I,Tsaoussoglou M, Panaghiotopoulou-Gar- 
tagani P, Chrousos G, Kaditis AG. Age-dependent changes in the size of adenotonsillar tissue in childhood: implications for sleep-disordered breathing. J Pediatr. 2013 Feb;162(2):269-74.e4.

4. Merati AL, Rieder AA. Normal endoscopic anatomy of the pharynx and larynx. Am J Med. 2003 Aug;115 Suppl 3A:10S-14S.

5. Handelman CS, Osborne G. Growth of the nasopharynx and adenoid development from one to eighteeen years. Angle Orthod. 1976 Jul;46(3):243-59.

6. Brodsky L. Modern assessment of tonsils and adenoids. Pediatr Clin North Am. 1989 Dec;36(6):1551-69.

7. Josephson GD, Duckworth L, Hossain J. Proposed definitive grading system tool for the assessment of adenoid hyperplasia. Laryngoscope. 2011 Jan;121(1):187-93.

8. Franco RA Jr, Rosenfeld RM, Rao M. First place: resident clinical science award 1999. Quality of life for children with obstructive sleep apnea. Otolaryngol Head Neck Surg. 2000 Jul;123(1 Pt 1):9-16.

9. Kumar DS, Valenzuela D, Kozak FK, Ludemann JP, Moxham JP, Lea $\mathrm{J}$, et al. The reliability of clinical tonsil size grading in children. JAMA Otolaryngol Head Neck Surg. 2014 Nov;140(11):1034-7.

10. Friedman M, Tanyeri H, La Rosa M, Landsberg R, Vaidyanathan K, Pieri S, et al. Clinical predictors of obstructive sleep apnea. Laryngoscope. 1999 Dec;109(12):1901-7.

11. Clemens J, McMurray JS, Willging JP. Electrocautery versus curette adenoidectomy: comparison of postoperative results. Int J Pediatr Otorhinolaryngol. 1998 Mar;43(2):115-22.

12. Fregosi RF, Quan SF, Kaemingk KL, Morgan WJ, Goodwin JL, Cabrera R, et al. Sleep-disordered breathing, pharyngeal size and soft tissue anatomy in children. J Appl Physiol (1985). 2003 Nov;95(5): 2030-8.

13. Brodsky L, Adler E, Stanievich JF. Naso- and oropharyngeal dimensions in children with obstructive sleep apnea. Int J Pediatr Otorhinolaryngol. $1989 \mathrm{Feb} ; 17(1): 1-11$.

14. Arens R, McDonough JM, Corbin AM, Rubin NK, Carroll ME, Pack
AI, et al. Upper airway size analysis by magnetic resonance imaging of children with obstructive sleep apnea syndrome. Am J Respir Crit Care Med. 2003 Jan;167(1):65-70.

15. Schwab RJ, Pasirstein M, Pierson R, Mackley A, Hachadoorian R, Arens $\mathrm{R}$, et al. Identification of upper airway anatomic risk factors for obstructive sleep apnea with volumetric magnetic resonance imaging. Am J Respir Crit Care Med. 2003 Sep;168(5):522-30.

16. Korhan I, Gode S, Midilli R, Basoglu OK. The influence of the lateral pharyngeal wall anatomy on snoring and sleep apnoea. J Pak Med Assoc. 2015 Feb;65(2):125-30.

17. Tauman R, GulliverTE, Krishna J, Montgomery-Downs HE, O’Brien LM, Ivanenko A, et al. Persistence of obstructive sleep apnea syndrome in children after adenotonsillectomy. J Pediatr. 2006 Dec; 149(6):803-8.

18. Ye J, Liu H, Zhang GH, Li P, Yang QT, Liu X, et al. Outcome of adenotonsillectomy for obstructive sleep apnea syndrome in children. Ann Otol Rhinol Laryngol. 2010 Aug;119(8):506-13.

19. Tagaya M, Nakata S, Yasuma F, Mitchell RB, Sasaki F, Miyazaki S, et al. Children with severe or moderate obstructive sleep apnoea syndrome show a high incidence of persistence after adenotonsillectomy.Acta Otolaryngol. 2012 Nov;132(11):1208-14.

20. Shintani T, Asakura K, Kataura A. The effect of adenotonsillectomy in children with OSA. Int J Pediatr Otorhinolaryngol. 1998 Jun;44(1): 51-8.

21. Abdel-Aziz M, Ibrahim N,Ahmed A, El-Hamamsy M,Abdel-Khalik MI, El-Hoshy H. Lingual tonsils hypertrophy; a cause of obstructive sleep apnea in children after adenotonsillectomy: operative problems and management. Int J Pediatr Otorhinolaryngol. 2011 Sep; 75(9):1127-31.

22. Schwengel DA, Sterni LM, Tunkel DE, Heitmiller ES. Perioperative management of children with obstructive sleep apnea. Anesth Analg. 2009 Jul;109(1):60-75. 